\title{
MESON PRODUCTION ON THE NUCLEON IN THE GIESSEN K-MATRIX APPROACH*
}

\author{
H. LENSKE, V. SHKLYAR, U. MOSEL \\ Institut für Theoretische Physik, Universität Giessen \\ Heinrich-Buff-Ring 16 \\ D-35392 Giessen, Germany \\ E-mail: horst.lenske@physik.uni-giessen.de
}

\begin{abstract}
Meson production on the proton is described in a coupled channels K-matrix approach. Both hadronic and photonic scenarios are taken into account on the same theoretical footing. At tree-level the Born amplitudes are obtained from an effective Lagrangian with phenomenologically adjustable coupling constants. The spectral distributions, resonances and their width and the background contributions are obtained within the same approach, thus accounting properly for interference effects. Applications to omega- and associated strangeness production on the proton are discussed.
\end{abstract}

\section{Introduction}

The nucleon as an entity of strongly interacting constituents is playing the gateway to low-energy QCD as realized in hadrons. Investigating its structure and dynamics by various probes and measuring a variety of observables will complete the much wanted data base on spectral properties of resonances, their excitations and decays, including information about branching ratios into the various meson-nucleon channels. An equally important motivation is the search for missing resonances trying to bridge the gap between the number of excited states of the nucleon predicted by quark models and the - at least until now - much fewer resonances seen in pion- or photon-induced reactions. In any respect, research on the nucleon structure will necessarily include the need for a good understanding of reaction dynamics. For that aim a realistic description is necessary, accounting properly for the interplay of various production channels, the interference among resonant and non-resonant parts of the scattering amplitudes. Ac-

*Work partially supported by FZ Jülich 
cepting this as a guide line, it is obvious that single channel descriptions in general will fail.

Coupled channel approaches are meeting these goals. They are able to account for the various aspects of meson-nucleon and photon-nucleon interactions and the cross talk between the various dynamical sectors. Only with such a more involve description we will be able to distinguish between the dynamical and the intrinsic, QCD related properties of spectral structures observed in cross sections. The Giessen model formulated a few years ago in ${ }^{1,2}$ and extended subsequently in ${ }^{3,4,5}$ is in line with these requirements. The model uses a unitary coupled-channel effective Lagrangian approach. It has been successfully applied in the analysis of pion- and photon-induced reactions in the energy region up to $2 \mathrm{GeV}$. The resonance couplings are simultaneously constrained by available experimental data from all open channels. While our previous analyses ${ }^{3,4}$ have been restricted to resonances with spin $J \leq \frac{3}{2}$ we have extended the description quite recently to higher spin states ${ }^{6}$, thus enlarging the model space and increasing the predictive power of the calculations. We now include essentially all channels contributing significantly to the cross sections in the energy region up to $2 \mathrm{GeV}$. The Giessen model is briefly summmarized in sect. 2. We then discuss recent applications to $\omega$-meson production in sect. 3 and to the associated strangeness production in sect. 4. The report closes with a short summary and outlook in sect. 5 .

\section{The Giessen Model: Coupled Channels K-Matrix Description of Meson Production}

The Giessen model describes meson production on the nucleon on the basis of an effective Lagrangian. At the energy scales considered here the appropriate degrees of freedom are the nucleons and hyperons from the basic $\mathrm{SU}(3)$ flavor octet and their excited states and, on the meson side, the pseudoscalar and vector meson octet states, supplemented by the photon and the electromagnetic coupling of the hadrons ${ }^{3}$. The principal structure of the model at tree-level is depicted in Fig.1. Here, we only briefly discuss the K-matrix part of the approach. The starting point is the decomposition of a Green function into a principal value and pole part given by a Dirac delta-function:

$$
G_{b s} \equiv \frac{P}{H-\omega}+i \pi \delta(H-\omega)
$$


With the propagators cast into this form the Bethe-Salpeter equation can be represented by a set of two coupled equations

$$
\begin{aligned}
\mathcal{K} & =\mathcal{V}+\mathcal{V} \frac{P}{H-\omega} \mathcal{K} \sim \mathcal{V} \\
\mathcal{M} & =\mathcal{K}+i \mathcal{K} \delta(H-\omega) \mathcal{M}
\end{aligned}
$$

where the first equation defines the K-matrix, corresponding for a Hermitian Born amplitude $\mathcal{V}=\mathcal{V}^{\dagger}$ to the real part of the scattering amplitude. The solution of the above equation for a multi-channel problem is a matrix containing the scattering amplitudes from channels $\alpha$ to channels $\beta$

$$
\mathcal{M}_{\alpha \beta} \equiv\left[\frac{\mathcal{K}}{1-i \mathcal{K}}\right]_{\alpha \beta} \sim\left[\frac{\mathcal{V}}{1-i \mathcal{V}}\right]_{\alpha \beta}
$$

where $\alpha$ and $\beta$ denote any of the photoproduction or hadronic production channels. The validity and quality of the K-matrix approach has been tested positively by various groups, e.g. ${ }^{7}$.

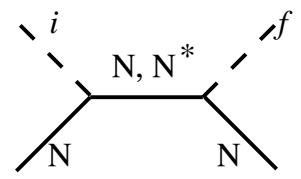

(a)

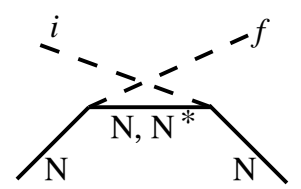

(b)

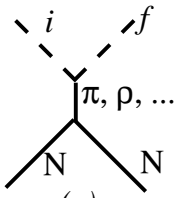

(c)

Figure 1. Born-diagrams in the $\mathrm{s}, \mathrm{u}$, and $\mathrm{t}$ channel contributing to the Bethe-Salpeter equation.

\section{3. $\omega$ Meson Production off the Nucleon}

In this section our primary interest is the $\omega$ meson production in $\pi p$ and $\gamma p$ reactions, as discussed in detail in ${ }^{8}$. Most of the theoretical studies of this reaction are based on a rather simplified single channel effective Lagrangian approach, e.g. 11,12. But there is agreement on the importance of the t-channel $\pi_{0}$-exchange contributions, which were studied by Friman and Soyeur ${ }^{13}$. However, the claims by the various models on the contributions of different resonances to the $\omega N$ final state are controversial ${ }^{8}$. Compared to our previous findings ${ }^{3,4}$ we observe significant changes by inclusion of spin $-\frac{5}{2}$ resonance contributions. To provide an additional constraint on the resonance couplings to $\omega N$ we also included the recent data on the spin density matrix obtained by the SAPHIR group ${ }^{10}$. 


\subsection{Hadronic Production: $\pi N \rightarrow \omega N$}

All experimental data on the $\omega$-meson production in the $\pi N$ scattering have been measured before 1980 and therefore have rather poor statistics. In total, there are 115 data points which includes differential and total cross sections data. The inclusion of spin- $\frac{5}{2}$ resonance contributions affects the $\omega N$ final state considerably. The main contributions close to the threshold come from the $P_{13}$ and $D_{15}$ partial waves. The resonance part of the production amplitude is dominated by the $D_{15}(1675)$ state $^{8}$. Overall, the inclusion of spin- $\frac{5}{2}$ resonances shifts strength to the $P_{13}$ and $D_{15}$ partial waves.

We also find strong contributions from the $P_{13}$ partial wave to the $\pi N \rightarrow \omega N$ reaction what has been already reported in ${ }^{3}$. The strength in this partial wave is shifted to the lower energies and becomes more pronounced at the reaction threshold. A peaking behavior seen in the $P_{13}$ partial cross section is due to the interference pattern between $P_{13}$ resonances and background contributions to the $\omega N$ channel. Hence, this is a representative example that the collaboration of resonance and background features can produce structures in cross sections which are easily misinterpreted as a resonance. Since the major contributions to the $\pi N \rightarrow \omega N$ reaction come from the $P_{13}$ and $D_{15}$ waves, it is interesting to look at the $\pi N$ inelasticity for these partial waves which are found in ${ }^{8}$. They lead to the conclusion that probably inelasticities from other channels, e.g. $(3 \pi N)$, should also be included.

\subsection{Photoproduction: $\gamma N \rightarrow \omega N$}

The differential $\omega$ meson photoproduction cross sections are presented in Fig.2. With the $\frac{5}{2}$ components included we obtain $\chi_{\gamma \omega}^{2}=4.5$ which significantly improves our previous result $\left(\chi_{\gamma \omega}^{2}=6.25\right)^{3,4}$. The strong $\pi^{0}$ exchange lead to a peaking behavior of the calculated differential cross sections at forward angles which are clearly visible in the SAPHIR measurements 10 above $1.783 \mathrm{GeV}$ and the theoretical cross sections, both displayed in Fig. 2. More detailed information of the production mechanism is obtained from observables measuring the spin degree of freedom of the $\omega$ meson. In the Gottfried-Jackson frame, where the initial photon and exchange particle are in their rest frame, and $z$-axis is in the direction of the incoming photon momentum, the calculation gives $\rho_{00}^{G J}=0$. The experimental value of $\rho_{00}^{G J}$ for forward directions, where the $\pi^{0}$ exchange dominates, was measured by SAPHIR and found to be in the range of $\rho_{00}^{G J}=0.2 \cdots 0.3$. Thus, 


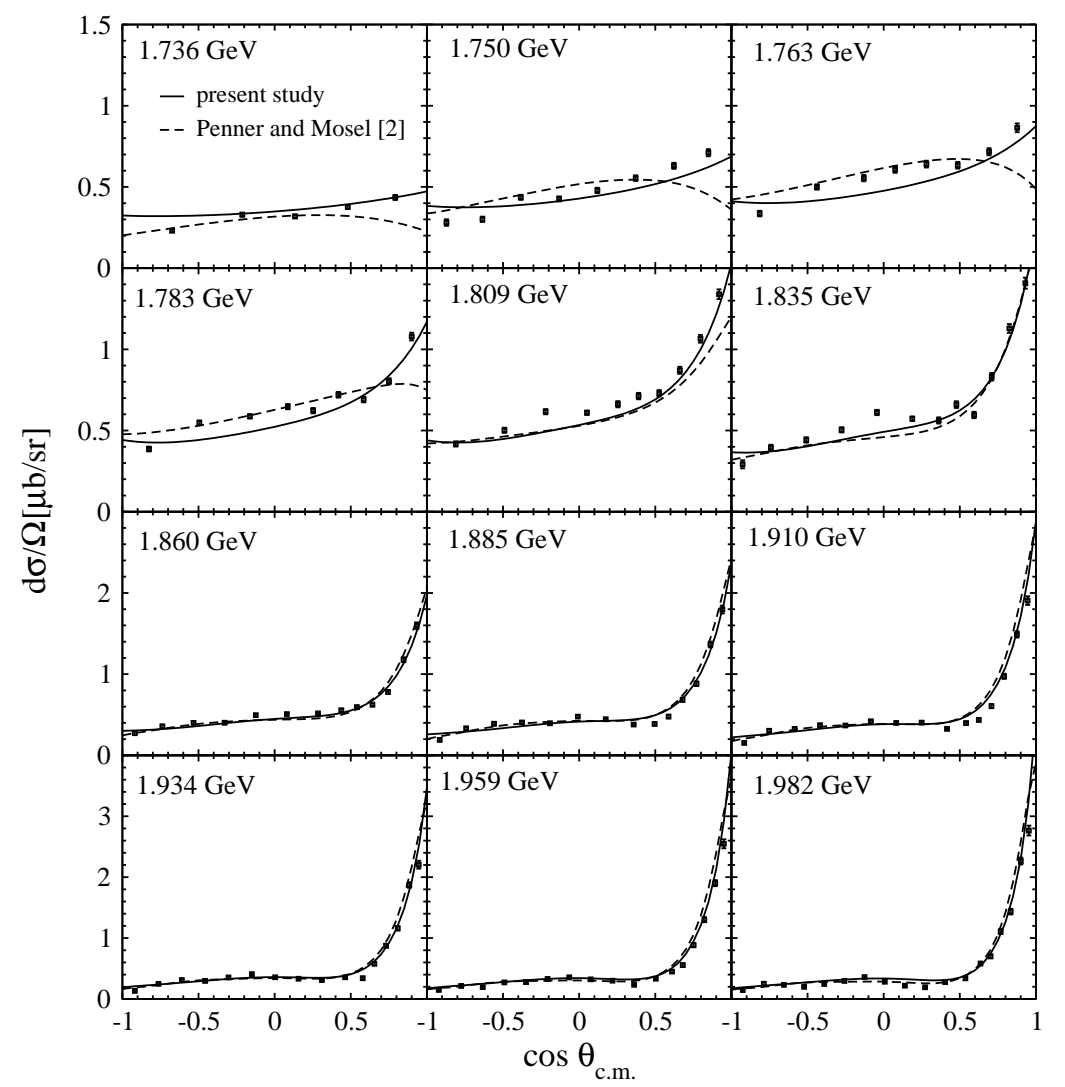

Figure 2. $\quad \gamma N \rightarrow \omega N$ differential cross sections in comparison with the SAPHIR data 10 and our previous results from ${ }^{4}$.

the nonzero matrix element testifies that even in this kinematical region other mechanisms (rescattering effects, interference with resonances) must be important.

Beside the $\pi^{0}$ exchange the largest contributions to $\omega$ meson photoproduction comes from the subthreshold spin- $\frac{5}{2}$ resonances: $D_{15}(1675)$ and $F_{15}(1680)$. Since the $\pi^{0}$ exchange above $1.8 \mathrm{GeV}$ strongly influences the $\gamma N \rightarrow \omega N$ reaction a consistent identification of individual resonance contributions from only the partial wave decomposition is difficult. The $P_{13}(1900)$, and $F_{15}(2000)$, and $D_{13}(1950)$ states which lie above the reaction threshold hardly influence the reaction due to their small couplings to $\omega N$. Despite of the small relative contribution from the $D_{15}$ and $F_{15}$ 

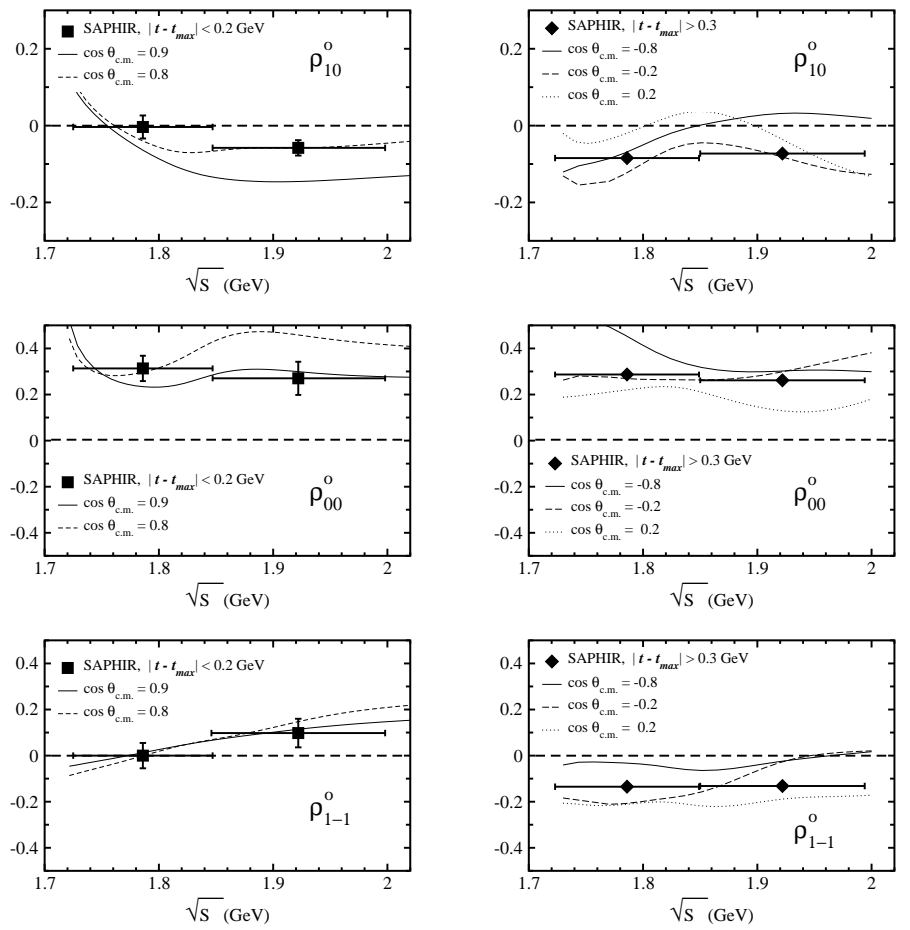

Figure 3. Spin density matrix elements in the helicity frame compared to the SAPHIR measurements ${ }^{10}$.

waves to the $\omega$ photoproduction the cross sections are strongly affected by spin- $\frac{5}{2}$ states because of the destructive interference pattern between the $\pi^{0}$ exchange and these resonance contributions.

While $F_{15}(1680)$ plays only a minor role in the $\pi N \rightarrow \omega N$ reaction the contribution from this state becomes more pronounced in the $\omega$ meson photoproduction because of its large $A_{\frac{3}{2}}^{p}$ helicity amplitude. The importance of the $F_{15}(1680)$ resonance to the $\omega$ meson photoproduction was also found by Titov and Lee ${ }^{12}$ and by Zhao ${ }^{15}$. However, in contrast to ${ }^{12}$ where also a large effect from $D_{13}(1520)$ was observed we do not find any visible contribution from this state. In fact, as discussed in ${ }^{8}$ a strong contribution found in the $D_{13}$ partial wave, resembling a resonance structure, comes in fact from non-resonant $\pi^{0}$ exchange.

The spin density matrix elements $\rho_{r r^{\prime}}$ extracted from the SAPHIR data ${ }^{10}$ are an outcome of the averages over rather wide energy and angle regions, 
see Fig. 3. The inclusion of measured $\rho_{r r^{\prime}}$ into the calculations provides a strong additional constraint on the relative partial wave contributions and finally on the resonance couplings. A satisfactory description of the spin density matrix is obtained in a wide energy region. Since the $\rho_{r r^{\prime}}$ data put strong constraints on the $\gamma p \rightarrow \omega p$ reaction mechanism there is an urgent need for precise measurements of the spin density matrix in more narrow energy bins to determine the reaction picture. Further details on beam asymmetries are found in ${ }^{8}$.

\section{Associated Strangeness Production on the Nucleon}

Since the recent $K \Lambda$ photoproduction data ${ }^{17,18}$ give an indication for 'missing' resonance contributions, a combined analysis of the $(\pi, \gamma) N \rightarrow K \Lambda$ reactions becomes inevitable to pin down these states. Assuming small couplings to $\pi N$, these 'hidden' states should not exhibit themselves in the pion-induced reactions and, consequently, in the $\pi N \rightarrow K \Lambda$ reaction. The decay ratios to the non-strange final states and the electromagnetic properties can be found in ${ }^{8}$. Our most recent results in the extended approach are given in ${ }^{9}$. In the that work, we have considered the partially contradicting CLAS and SAPHIR data separately by performing independent fits to either of the two data sets. In the following, the corresponding results are denoted by the indices $C$ and $S$, respectively.

\subsection{Hadronic Strangeness Production: $\pi N \rightarrow K \Lambda$}

The $S$ - and $C$ - calculations differ in their description of the non-resonance couplings to $K \Lambda$. As a consequence, different background strengths are obtained for the $S_{11}, P_{11}$, and $P_{13}$ partial waves while leaving the $P_{13}(1720)$ and $P_{13}(1900)$ resonance couplings almost unchanged ${ }^{9}$. Comparing the $S$ and $C$-parameter sets, the largest difference in the resonance parameters is observed for the $P_{11}(1710)$ state. This resonance is found to be almost completely of inelastic origin with a small branching ratio to $\pi N^{9}$. This state gives only a minor contribution to the reaction and the observed difference in the $P_{11}$ partial wave between $S$ - and $C$-results is due to the Born term and the $t$-channel exchange contributions.

The calculated differential cross sections corresponding to the $S$ - and $C$-coupling sets are found in ${ }^{9}$. Both results show a good agreement with the experimental data in the whole energy region. A difference between the two solutions is only found at forward and backward scattering angles. This is due to the fact that the CLAS photoproduction cross sections rise at 
backward angles which is not observed by the SAPHIR group (see discussion below). At other scattering angles the $S$ and $C$ results are very similar. The differences between $S$ - and $C$-calculations are more pronounced for the $\Lambda$-polarization. Again, the main effect is seen at the backward angles where the polarization changes its sign in the $C$-calculations. Unfortunately, the quality of the data does not allow to determine the reaction mechanism further.

\subsection{Photoproduction of Strangeness: $\gamma N \rightarrow K \Lambda$}

The older SAPHIR measurements ${ }^{16}$ show a resonance-like peak in the total photoproduction cross section around $1.9 \mathrm{GeV}$. The more recent SAPHIR 17 and CLAS ${ }^{18}$ data confirm the previous findings. However, the interpretation of these data is controversial leaving open questions whether in these measurements contributions from presently unknown resonances are observed or if they can be explained by already established reaction mechanisms.

Guided by the results of ${ }^{4}$ we have performed a new coupled-channel study of this reaction using separately the CLAS and SAPHIR measurements as two independent input sets. The main difference between the CLAS and SAPHIR data is seen at backward and forward directions, Fig. 4. Both measurements show two peaks but disagree in the absolute values of the corresponding differential cross sections. Also, the second bump in the CLAS data is shifted to the lower energy $1.8 \mathrm{GeV}$ for the scattering angles corresponding to $\cos \theta=0.35$ and $\cos \theta=0.55$.

Similar to $\pi N \rightarrow K \Lambda$ the major difference between the $S$ and $C$ solutions is the treatment of the non-resonant contributions. As seen in Fig. 4, both calculations show two peak structures in the differential cross sections at 1.7 and $1.9 \mathrm{GeV}$. In both cases the first bump at $1.67 \mathrm{GeV}$ is produced by the $S_{11}(1650)$ resonance. The relative contributions to the second peak at $1.9 \mathrm{GeV}$ are different in the $C$ and $S$ solutions. In the $C$-calculations this structure is described by the $S_{11}$ partial wave. At higher energies the $S_{11}$ channel is dominated by the non-resonant reaction mechanisms and there is no need to include a third $S_{11}$ resonance, as done e.g. in ${ }^{20}$. The $P_{13}$ partial wave is entirely driven by the $P_{13}(1720)$ and $P_{13}(1900)$ resonance contributions. Switching off these resonance couplings to $K \Lambda$ leads to an almost vanishing $P_{13}$ partial wave. In the $S$-calculations no peaking behavior is found in the $S_{11}$ partial wave at $1.95 \mathrm{GeV}$. However, the non-resonant effects in the $S_{11}$ channel are still important. The role of the $P_{13}$ resonances 


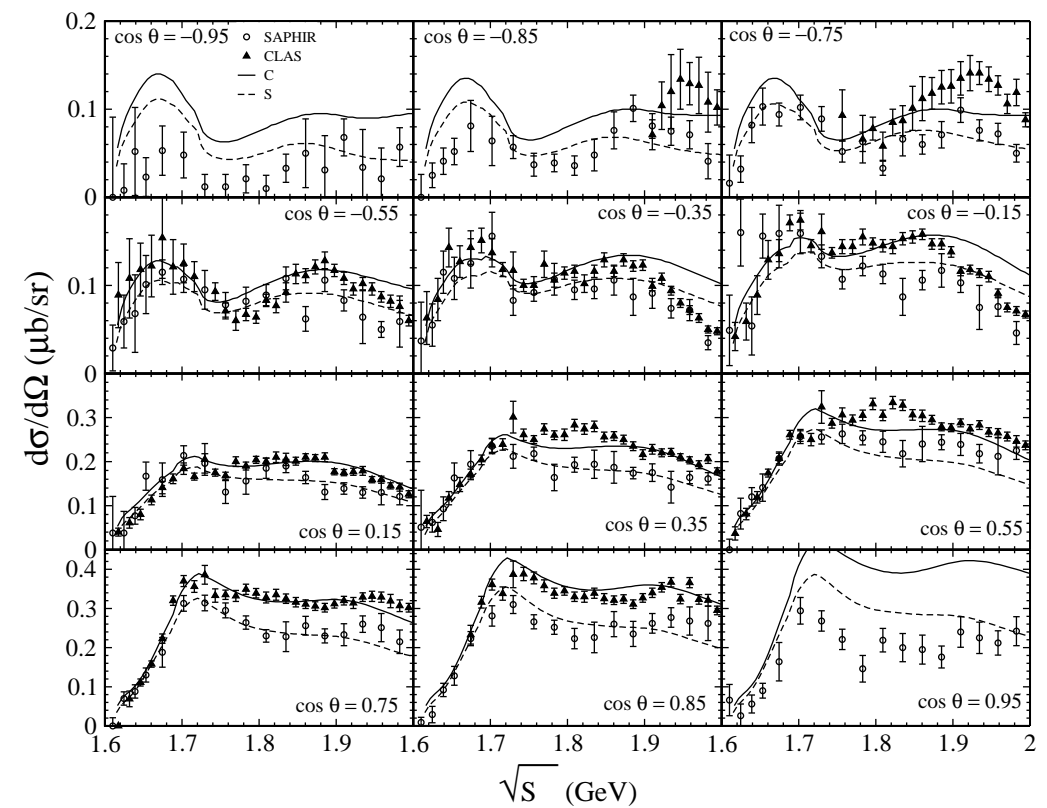

Figure 4. Comparison of the differential cross sections for the reaction $\gamma p \rightarrow K^{+} \Lambda$ calculated using $C$ and $S$ parameter sets. Experimental data are taken from ${ }^{18}$ (CLAS) and ${ }^{17}$ (SAPHIR).

are slightly enhanced in the $S$-calculations. The effect from the $P_{11}(1710)$ resonance is found to be small in both calculations due to destructive interference with the background process. There are no significant contributions from the spin- $\frac{5}{2}$ resonances to the $\gamma N \rightarrow K \Lambda$ reaction.

The calculated photon beam asymmetry $\Sigma_{x}$ and recoil polarization $P_{\Lambda}$ are shown in Fig. 5 and Fig. 6. Since the beam asymmetry data from the SPring- 8 collaboration ${ }^{21}$ are available only for energies above 1.94 $\mathrm{GeV}$, these measurements give an insignificant constraint on the model parameters. Therefore, the results for the asymmetry might be regarded as a prediction rather than an outcome of the fit. More information comes from the $\Lambda$-polarization data. A good description of the $\Sigma_{x}$ and $P_{\Lambda}$ data is possible in both the $C$ and $S$ calculations. 


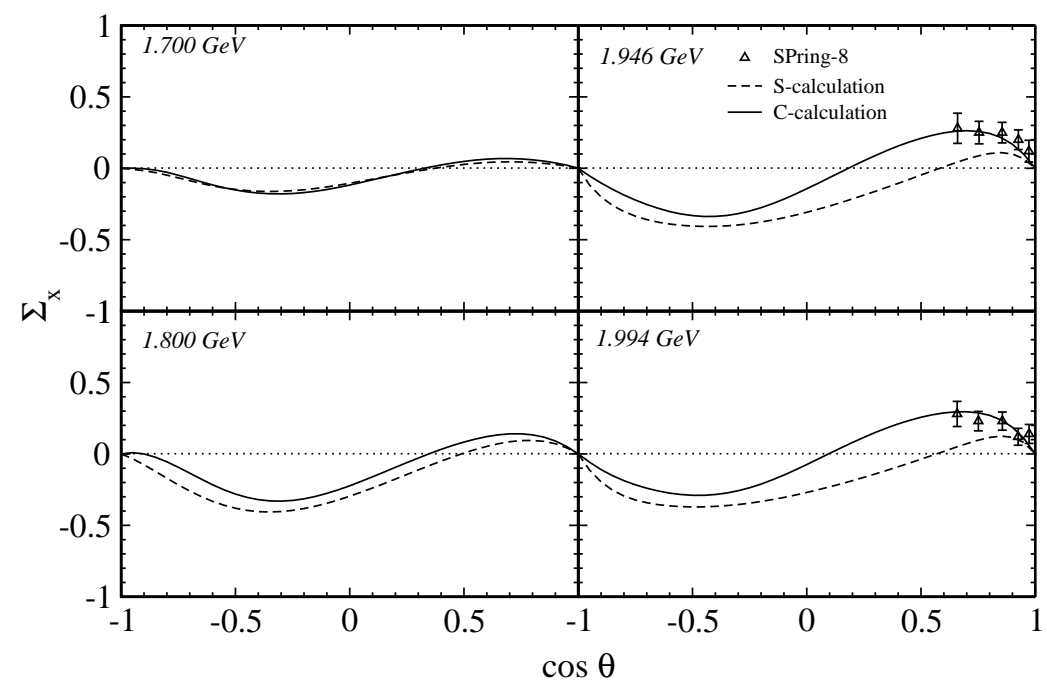

Figure 5. The calculated photon beam asymmetry. Data are taken from ${ }^{21}$

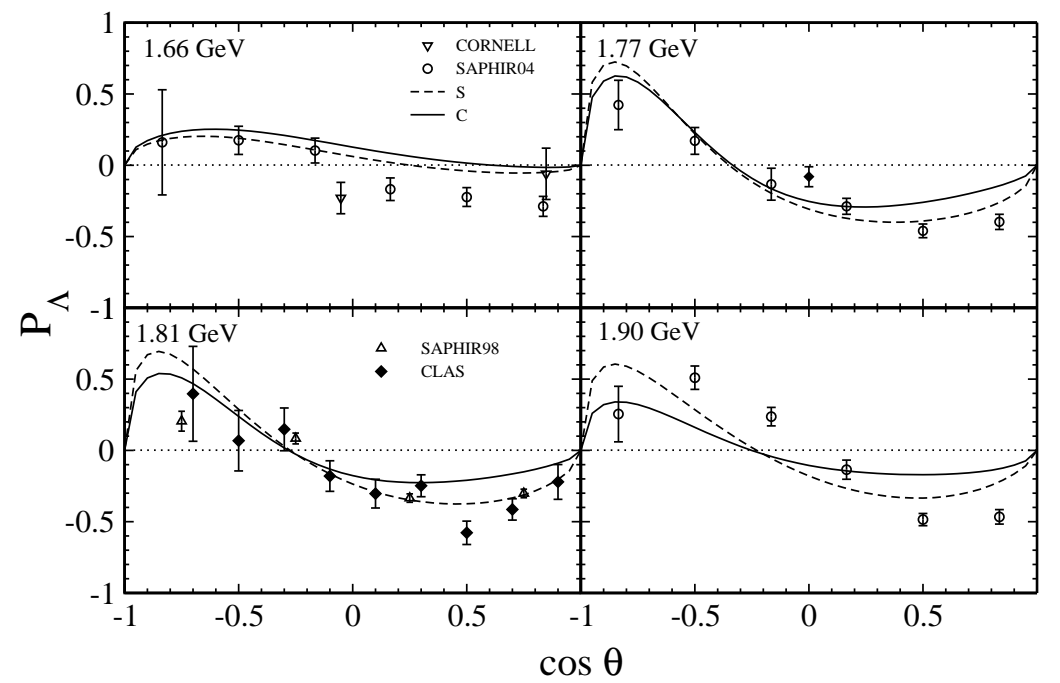

Figure 6. $\Lambda$-polarization in the $\gamma p \rightarrow K^{+} \Lambda$ reaction. Data are from SAPHIR98 ${ }^{16}$, SAPHIR0 ${ }^{17}$, CLAS ${ }^{18}$, CORNELL ${ }^{19}$.

\section{Summary and Outlook}

The importance of a controlled treatment of channel coupling for a quantitative understanding of meson production on the nucleon was pointed out. 
An approach, fulfilling the - sometimes delicate - balance between flexibility and generality, is given by using a Lagrangian model in conjunction with a decent reaction theory. Such a programm is underlying the Giessen model, describing meson production by a coupled channels K-matrix approach, based on a Lagrangian with phenomenological coupling constants and from factors.

The results for $\omega$ meson production and associated strangeness production by $K \Lambda$ processes are convincing in their ability to describe various experimental data, from total and differential cross sections to spin observables. The close connection between hadronic and photonic production channels was discussed for the $\omega N$ reaction. In both $\omega N$ and $K \Lambda$ reactions the importance of a dynamical treatment of the reaction mechanism as in the Giessen model was evident by the fact that come of the spectral structures were due to quantum mechanical interference phenomena. In order to resolve those effects also in the experimental data, measurements of spin observables play a crucial role.

\section{References}

1. T. Feuster and U. Mosel, Phys. Rev. C58, 457 (1998), nucl-th/9708051;

2. T. Feuster and U. Mosel, Phys. Rev. C 59, 460 (1999), nucl-th/9803057.

3. G. Penner and U. Mosel, Phys. Rev. C 66, 055211 (2002), nucl-th/0207066.

4. G. Penner and U. Mosel, Phys. Rev. C 66, 055212 (2002), nucl-th/0207069.

5. G. Penner and U. Mosel, Phys. Rev. C 65, 055202 (2001), nucl-th/0111023.

6. V. Shklyar, G. Penner, and U. Mosel, Eur. Phys. J. A21, 445 (2004), nuclth/0403064.

7. T. Sato and T. S. H. Lee, Phys. Rev. C54, 2660 (1996), nucl-th/9606009.

8. V. Shklyar, H. Lenske, and U. Mosel, Phys. Rev. C 71, 055206 (2005).

9. V. Shklyar, H. Lenske, and U. Mosel, Phys. Rev. C 72, 015210 (2005)

10. J. Barth et al., Eur. Phys. J. A 18, 117 (2003).

11. Q. Zhao, Z. Li, and C. Bennhold, Phys. Rev. C 58, 2393 (1998).

12. A. I. Titov and T. S. H. Lee, Phys. Rev. C 66, 015204 (2002).

13. B. Friman and M. Soyeur, Nucl. Phys. A600, 477 (1996).

14. Y. Oh, A. Titov, and T. S. H. Lee, Phys. Rev. C 63, 025201 (2001).

15. Q. Zhao, Phys. Rev. C63, 025203 (2001), nucl-th/0010038.

16. M. Q. Tran et al., Phys. Lett. B445, 20 (1998).

17. K. H. Glander et al., Eur. Phys. J. A19, 251 (2004), nucl-ex/0308025.

18. J. W. C. McNabb et al., Phys. Rev. C69, 042201 (2004), nucl-ex/0305028.

19. H. Thom, E. Gabathuler, E. Jones, B. McDaniel, and W. M. Woodward, Phys. Rev. Lett. 11, 433 (1963).

20. B. Julia-Diaz et al., (2005), nucl-th/0501005.

21. R. G. T. Zegers et al., Phys. Rev. Lett. 91, 092001 (2003), nucl-ex/0302005. 